\title{
Las experiencias profesionales de los estudiantes de interpretación como fuente de mejora en los programas de formación de intérpretes en Chilel
}

\author{
Pedro Pavez-Gamboa \\ pedro.pavez@pucv.cl \\ Pontificia Universidad Católica de Valparaíso, Valparaíso, Chile
}

\author{
Ámbar Urra-Urtubia \\ ambar.v.urra@gmail.com \\ Universidad de Las Américas, Viña del Mar, Chile
}

\author{
Carol Carvajal-Pallacán \\ carol.carvajal.p@gmail.com \\ Universidad de Las Américas, Viña del Mar, Chile
}

\begin{abstract}
Resumen
Existe un consenso general respecto a que la experiencia en el campo profesional, previo al término del proceso formativo, es crucial no solo para una transición más adecuada al campo laboral, sino también para promover el desarrollo integral del estudiante. Las actividades profesionales que los estudiantes realizan se convierten en mediaciones privilegiadas del proceso de formación de los profesionales. Por ello, la presente investigación se centra en alumnos de interpretación que tuvieron experiencias profesionales durante su formación. En este contexto, la consideración de las voces de los estudiantes es crucial para identificar nuevas necesidades en la formación de intérpretes. Mediante entrevistas a quince estudiantes con experiencia profesional de tres universidades de Chile, se indagó en las percepciones y expectativas que estos tienen respecto a la experiencia profesional vivida y su formación. De este modo se determinó que la experiencia profesional previa al egreso permite la incorporación de nuevos conocimientos, acciones, técnicas y actitudes, así como también facilitan la resignificación del proceso de aprendizaje y enseñanza, transformándolo en un proceso bidireccional, en la que las experiencias de los estudiantes mejoran los programas formativos.
\end{abstract}

Palabras clave: formación de intérpretes, experiencia profesional, voces de los estudiantes, educación dialógica, percepción.

1 Artículo realizado a partir de la investigación "Las experiencias profesionales de los estudiantes de interpretación como fuente de mejora en los programas de formación de intérpretes", llevada a cabo entre mayo y diciembre de 2017, con el apoyo y el aval de la Pontificia Universidad Católica de Valparaíso. 


\section{The Professional Experiences of Interpretation Students as a Source of Improvement on Interpreter Training Programs in Chile}

\section{Abstract}

There is general consensus that experience in the professional field, prior to the end of the training process, is crucial not only for a more adequate transition to working life, but also in promoting student's comprehensive development. Professional activities carried out by students become privileged mediations in their training process. Therefore, this research focuses on interpretation students who had professional experiences during their training. In this context, the consideration of students' voices is crucial to identify new needs in interpreter training. Fifteen interviews were conducted among students from three universities in Chile, who had had previous professional experiences. Their perceptions and expectations regarding their professional experience and their training were studied. Thus, it could be established that professional experience prior to graduation provide new knowledge, actions, techniques and attitudes, as well as facilitating the resignification of the learning and teaching process, by turning it into a bidirectional process, in which students' experiences help improve training programs.

Keywords: interpreter training, professional experience, students' voices, dialogic education, perception.

\section{La pratique professionnelle des étudiants d'interprétation comme démarche d'amélioration des programmes de formation d'interprètes au Chili}

\section{Résumé}

Il y a un consensus général que la pratique professionnelle sur le terrain, avant l'achèvement du processus d'apprentissage, est cruciale non seulement pour une transition plus pertinente au marché du travail, mais aussi dans la promotion du développement intégral des étudiant(e)s. Les pratiques professionnelles sur le terrain deviennent des médiations privilégiées du processus de formation. Dans cette optique, la présente recherche est axée sur les étudiants d'interprétation qui ont eu une expérience professionnelle au cours de leur formation. Dans ce contexte, il s'avère crucial de tenir en compte les opinions des étudiants pour identifier les nouveaux besoins en matière de formation des interprètes. Quinze entretiens auprès des étudiant(e)s ayant eu une expérience professionnelle dans le marché du travail ont été menés dans trois universités chiliennes. Nous avons enquêté sur les perceptions et les attentes que ces étudiant(e)s ont eu sur leurs expériences dans le marché du travail et sur leur formation. En guise de conclusion, nous avons trouvé que les pratiques professionnelles préalables à l'achèvement des études universitaires permettent non seulement d'incorporer de nouvelles connaissances, des actions, des techniques et des attitudes, mais permettent aussi de faciliter la re-signification du processus d'enseignement et d'apprentissage en le transformant en un processus à double sens, dont les expériences professionnelles des étudiants servent à améliorer les programmes de formation

Mots-clés : Formation d'interprètes, pratique professionnelle sur le terrain, opinion des étudiant(e)s, éducation dialogique, perception. 


\section{Introducción}

La educación superior cambia incesantemente. Hoy por hoy se entiende que la enseñanza y el aprendizaje van de la mano con la concepción de una universidad más dinámica y eficiente, especialmente por la relación que guardan los centros académicos con el mundo profesional. En este contexto, la transición entre la educación superior y el mercado laboral puede ser un punto conflictivo en la trayectoria profesional y también en la vida del estudiante (Correa y Pereira, 2013). La cantidad de estudiantes universitarios ha aumentado en la mayoría de los países de la OCDE (OCDE, 2005). Al mismo tiempo, la transición entre la universidad y el mundo profesional se ha hecho más compleja, pues nuevas cohortes de estudiantes pueden enfrentarse a mayores dificultades en procesos de inserción laboral mucho más largos y difusos de lo que solían ser (Salas-Velasco, 2007).

Así, la estructura de los programas y enfoques formativos de la educación universitaria pasan actualmente por un intenso debate y reflexión, debido al replanteamiento del rol de la educación superior en la sociedad (Zabalza, 2009). En este proceso, las características de los procesos formativos han ido variando, dejando atrás el paradigma centrado en el profesor para adoptar uno basado en el supuesto de que solo se logra un aprendizaje significativo cuando el estudiante asume la responsabilidad en la organización y desarrollo de su trabajo. Junto a esto, las universidades no pueden ser indiferentes al destino laboral de sus egresados, sino que deben hacerse cargo de que sus estudiantes desarrollen el dominio del saber, del saber hacer, del saber convivir y del saber ser (Jabif, 2007), no solo durante su proceso de formación, sino también en su carrera profesional.

Considerando esto último, las universidades han facilitado la transición hacia el mercado laboral mediante las prácticas profesionales, en el entendido de que el aprendizaje y el desarrollo de competencias no ocurren solo en el aula, sino también en un contexto profesional real. Sin embargo, al entender la universidad como un espacio social, donde el avance en la calidad depende de la institución misma, son los mismos actores de esta comunidad de aprendizaje y enseñanza quienes guiarán este cambio hacia un nuevo paradigma de la educación superior. En otras palabras, todo proceso de cambio está mediado por la capacidad de sus actores para integrar armónica y diferencialmente los componentes involucrados (Bovill, Cook-Sather y Felten, 2011).

En este contexto, las voces de los estudiantes se configuran como un elemento primordial para estimular y generar cambios socialmente validados en el proceso de formación. Si bien existen todavía muchos modelos de desarrollo profesional universitario que operan sobre la base de que los estudiantes no tienen, o no debiesen tener, un rol activo (Cook-Sather, 2007), hay evidencia suficiente para señalar que las colaboraciones con los estudiantes en todo proceso de cambio o mejora de los diferentes elementos de la educación superior tiene efectos positivos en la cocreación de procesos de enseñanza, del diseño de cursos y de currículos, lo que constituye una evidencia de que los estudiantes no solo cuentan con una nueva perspectiva respecto a su aprendizaje, sino que también tienen un entendimiento profundo a ese respecto (Bovill, Cook-Sather y Felten, 2011; Coates, 2005).

En vista de lo anterior, esta investigación da cuenta de las percepciones y expectativas de un grupo de estudiantes de interpretación respecto a su proceso de formación, recogidas a través de entrevistas semiestructuradas. La particularidad de estos quince estudiantes, de tres universidades diferentes estriba en que se 
encuentran ad portas de comenzar la transición hacia el mercado laboral, pero ya tuvieron la posibilidad de trabajar como intérpretes en diferentes entornos. Esa experiencia profesional en el campo de la interpretación puede dar nuevas luces respecto a la formación de intérpretes, dado que este tipo de experiencias demanda integrar el conocimiento aprendido en el aula y aplicarlo en un contexto laboral, lo que estimulará el desarrollo y la consolidación de saberes y habilidades (Zabalza, 2011).

El presente trabajo se organiza en cinco secciones: un marco teórico centrado en la formación de intérpretes y su relación con la experiencia profesional y la educación dialógica, que apunta a dar voz a los estudiantes respecto a su propia formación. A continuación, se describe la metodología de este estudio para finalizar con los resultados, su discusión y las conclusiones. La experiencia profesional como eje fundamental en la incorporación de nuevos conocimientos, acciones, técnicas y actitudes y como un elemento que resignifica el proceso de aprendizaje y enseñanza es la pregunta que subyace a la investigación.

\section{Marco teórico}

\subsection{La formación de intérpretes}

La enseñanza de la interpretación, como la conocemos en la actualidad, comenzó después de los acontecimientos históricos del siglo $\mathrm{xx}$, ya que fueron estos hechos los que impulsaron el desarrollo de la profesión como tal. De hecho, la Conferencia de la Paz de París se entiende como el hito fundacional de la profesión debido al aumento en la demanda de profesionales (Kalina, 2005), mientras que el origen de la interpretación simultánea fue fruto de las circunstancias lingüísticas que rodearon la Segunda Guerra Mundial (Driesen, 1999; Domínguez, 2016).
Según Domínguez (2016), la primera escuela de intérpretes data del año 1930 en Mannheim, en la Universidad de Heidelberg, considerada una de las más antiguas de Europa, junto con las escuelas de Viena y Ginebra, que surgieron una década más tarde. Asimismo, en la década de los 50 aparecieron fundaciones internacionales y profesionales, dentro de las cuales se destaca la Asociación Internacional de Intérpretes de Conferencias (AIIC), que apunta a la regularización y promoción de la profesión. Paralelamente, surgieron las primeras contribuciones y reflexiones respecto a los preceptos teóricos de la interpretación. De igual modo, se desarrollaron los primeros planes de estudios, que apuntaban a dar una explicación a los fenómenos relacionados con la interpretación y las metodologías de sus procesos de formación. E1 primer curso de interpretación, organizado por la OIT en 1928, establecía que la única manera de aprender era mediante la práctica constante (Baigorri, citado por Domínguez, 2015; Orlando, 2016; Pöchhacker, 2004), dinámica que se mantiene en la mayoría de los programas de formación de intérpretes (Gile, 2005), en los que se ejecutan acciones deliberadas que apuntan a la superación de dificultades y al logro de objetivos específicos, intentando reproducir las exigencias de una situación de interpretación real establecida por un profesor/intérprete experto que transmite sus conocimientos y experticia a los estudiantes.

En este período, la noción de que el intérprete era una persona con facultades innatas estaba bastante extendida (Mackintosh, 1999), pero esa idea del innatismo de la interpretación comenzó a ser desmentida por los cursos de formación de intérpretes, que demostraron que el intérprete podía formarse. Al mismo tiempo, el surgimiento y la consolidación de los estudios de traducción e interpretación como disciplina dieron paso a conocimientos que permitieron complementar la formación, como 
las tipologías de error (Barik, 1971), la teoría del sentido (Seleskovitch, 1975) y el modelo de esfuerzos (Gile, 1995).

Así, los estudios de interpretación, como interdisciplina de los estudios de traducción, complementan o critican las teorías existentes, apuntando a una investigación con aplicaciones didácticas a partir de disciplinas como la psicología, el análisis del discurso o la neurociencia (Domínguez, 2015). El interés por la pedagogía en interpretación creció hacia finales del siglo Xx y comienzos del XxI, lo que se manifestó en la publicación de monografías y manuales (Gi1lies, 2005; Jones, 1998; Collados y Fernández, 2001; Pöchhacker, 2004; Jiménez, 2012). Tal como señala Iglesias (citado por Domínguez, 2015), la investigación y su aplicación didáctica han contribuido a cimentar la metodología de la enseñanza de la interpretación en criterios más sistemáticos y rigurosos que dejan de lado intuiciones y experiencias personales y apuntan a la consolidación de la disciplina.

En este contexto, la formación de intérpretes se encuentra bajo corrientes de cambio a diferentes niveles. Si bien la enseñanza de la interpretación suele estar sujeta a cambios enmarcados en el cambio de paradigma en la educación superior (UNESCO, 1998; Kiraly, 2000), consistente en un proceso de aprendizaje y enseñanza centrado en el diálogo y en el estudiante, más que en un profesor/experto.

En Chile, Díaz-Galaz (2017) entrega un panorama bastante acabado de la enseñanza de la interpretación. La formación de intérpretes en este país comienza a finales de la última década del siglo $\mathrm{xx}$, cuando las instituciones de educación superior comenzaron a desarrollar sus programas de pregrado. Actualmente existen diecinueve de estos programas con más de 1.800 estudiantes. Vale destacar, que de esos programas solo cuatro ofrecen énfasis en la en- señanza de la interpretación. El resto entrega el título general de traductor-intérprete. Estos programas duran en promedio entre ocho y nueve semestres, y se centran en su mayoría en interpretación consecutiva y simultánea. Tradicionalmente, la enseñanza de la interpretación ha seguido las prácticas europeas, con enfoques de enseñanza impartida por intérpretes que se basan en su experiencia profesional y en su intuición pedagógica. Sin embargo, hoy en día se ha avanzado hacia una profesionalización de la docencia en interpretación, con iniciativas como la red nacional de formadores de traductores e intérpretes, junto con un número de seminarios y conferencias dedicadas al tema.

\subsection{Cambio de paradigma en la educación superior}

Ya no es novedad mencionar que la educación superior está sujeta a muchas dinámicas de cambio. Actualmente las universidades se abren a la sociedad que las acoge y les exige profesionales idóneos. Esto lo señala la UNESCO, cuando afirma que la formación de profesionales eficientes, éticos y socialmente responsables constituye la misión esencial de la educación superior contemporánea (UNESCO, 1998).

Al mismo tiempo, se hace referencia a la forma como las universidades imparten sus procesos formativos, junto con la magnitud en la que contribuyen a disminuir la brecha entre la adquisición del conocimiento y la capacidad de aplicarlo (Jabif, 2007). Esto incluye la demanda a las universidades de no ser indiferentes al destino laboral de sus egresados, pues limitarse a la transferencia de contenidos no es suficiente. Como establece Delors (1996), las universidades deben hacerse cargo de que los estudiantes desarrollen un dominio del saber, del saber hacer, del saber convivir y del saber ser (Jabif, 2007) no solo durante su proceso de formación, sino también en un pro- 
ceso de educación continua. En este sentido, De Miguel (2007) sostiene que en la sociedad del conocimiento es necesario aprender continuamente con el fin de comprender y dar respuesta a las nuevas situaciones emergentes, debido a que solo el desarrollo de la capacidad individual de autoaprendizaje permite entender y adaptarse a las exigencias de un mundo en constante cambio. Así, recalcamos, las metodologías de enseñanza y los currículos del siglo XXI deben asumir este principio como criterio fundamental. Este principio es reconocido incluso por organismos multilaterales; la OCDE (2012) señala que la capacidad de los estudiantes para potenciar la calidad de la educación superior es considerable si se les otorgan las herramientas adecuadas.

De un enfoque que limitaba el aprendizaje a la retención de información, a la escucha pasiva y a la observación, con un sistema de evaluación centrado en el resultado y netamente cuantitativo, se ha dado paso a un paradigma de carácter cualitativo, formativo y formador centrado en un modelo orientado al aprendizaje dialógico y colaborativo. Es decir, el proceso de enseñanza y aprendizaje debe construirse entre todos sus miembros (Zabalza, 2009), considerando todas las voces. El movimiento Student Voices emerge en este contexto como una manera de armonizar los diferentes elementos de la educación dialógica y la calidad en los procesos de aprendizaje y enseñanza. Se trata de una teoría y un conjunto de prácticas que posiciona al estudiante como agente activo de análisis y evaluación de sus propios procesos de aprendizaje (Bovill, Cook-Sather y Felten, 2011). Esto se ha adoptado en diferentes disciplinas, promoviendo el desarrollo de currículos (Mihans, Long y Felten, 2008), de estrategias de enseñanza y de evaluación pedagógica (Bovill, Cook-Sather y Felten, 2011), prácticas que sin duda pueden aplicarse en el contexto de la formación de intérpretes.
Kiraly (2000), por ejemplo, sostiene que es necesario contar un enfoque socioconstructivista en la enseñanza de la traducción e interpretación, debido a que el aprendizaje no es solamente una actividad individual, sino que es un proceso cognitivo interno y externo, colaborativo y cooperativo, parte de un fenómeno social. Al mismo tiempo señala que el aprendizaje significativo y el conocimiento se genera en interacciones intersubjetivas, en las que el lenguaje sirve como medio para resolver diferencias y alcanzar consensos. Por ende, este nuevo paradigma, de la mano con una educación socioconstructivista, tiene profundas implicaciones en la formación de intérpretes.

Pese a esto, los estudios sobre enseñanza de la interpretación llevados a cabo con estudiantes como sujetos de experimentos relacionados con la enseñanza de la misma han abordado temáticas como la evaluación, las aptitudes y metodologías de enseñanza y aprendizaje (Takeda, 2010). Si bien el rol del intérprete en formación ha sido abordado desde diferentes aristas, como la evaluación para el aprendizaje (Domínguez, 2015), o la metacognición y la autoevaluación (Choi, 2004), la investigación sobre las perspectivas de los estudiantes respecto a su propio proceso de aprendizaje sigue siendo un campo en expansión (Lee, 2005). Por ello, el presente trabajo se posiciona como una iniciativa que, tomando experiencias de otras disciplinas, recoge las voces de los estudiantes a modo de estimular la reflexión de formadores respecto a sus propias prácticas pedagógicas con el fin de mejorarlas.

\subsection{Experiencia profesional}

Como ya se señaló, los estudios de interpretación han abordado la formación de intérpretes desde diversos puntos de vista, apo- 
yados, por ejemplo, en la práctica constante para estimular el desarrollo de habilidades, contenido teórico y reflexión con el objetivo de generar aprendizajes colaborativos y cooperativos. Estos son componentes comunes en la mayoría de los programas (Gile, 2005; Kiraly, 2000). Junto a esto, es importante mencionar que a pesar de que existen factores en común en los programas de formación, se presenta también un variado rango de posibilidades y diferencias que dependen de los contextos locales dentro de los cuales se desarrollan (Gile, 2005).

El mercado laboral de estos contextos locales moldea hasta cierto punto los requerimientos en la formación a nivel superior, generando una relación de intermediación entre la demanda y la oferta mediante un enfoque formativo centrado en el aprendizaje, desarrollando así actividades académicas vinculadas con su entorno, con una docencia que articula el aula con los espacios profesionales sobre la base de la investigación y la extensión (Mungaray, 2001). De este modo, los criterios del mercado laboral y de la educación superior se abordan directamente en diseños curriculares consensuados entre todos los actores involucrados.

Actualmente, el constante intercambio de personas, bienes y servicios genera una demanda de comunicación de alto nivel a tal punto que la industria de la traducción e interpretación seguirá en aumento. A modo de ejemplo, el Department of Labor de Estados Unidos estima que el crecimiento de la industria en el mercado de la interpretación aumentará aproximadamente un 29\% entre el 2014 y el 2024 (Cabrera, 2017). Por ello, se requieren profesionales competentes lingüística y culturalmente hablando (Orlando, 2016).
Junto a lo anterior, en la interpretación, la empleabilidad de los egresados requiere de un trabajo de desarrollo de habilidades e incluso especialización (Alvarez-Álvarez y Arnáiz-Uzquiza, 2017), ya que se trata de una profesión mayoritariamente freelance o independiente (Olohan, citado por Olalla-Soler, 2018) por lo que la relación entre la formación y el mercado es cada vez más estrecha debido a que no hay una empresa empleadora de por medio entre el intérprete y el cliente final. Tal como señalan Walker y Shaw (2011), la brecha existente entre la formación de intérpretes y el mundo laboral ha sido reconocida y analizada. De hecho, ya en 1990, Resnick sugirió que iniciativas como las prácticas profesionales, las mentorías y una supervisión extendida podían mitigar la ya prolongada dificultad de preparar intérpretes dentro de un contexto académico para satisfacer las demandas del campo (Walker y Shaw, 2011). Esta transición es un fenómeno complejo que ha adquirido relevancia tanto en foros académicos como en los debates de políticas educativas, generando una tensión entre la academia, que apunta a la búsqueda del conocimiento, y la inmersión total en el mundo laboral, donde el conocimiento se traduce en acción profesional (Schnell y Rodríguez, 2017).

Los estudios de interpretación han abordado la cuestión de la empleabilidad de los intérpretes en sus múltiples dimensiones. Por ejemplo, en el contexto institucional (Ruiz y Diur, 2017); desde la perspectiva de los empleadores (Schnell y Rodríguez, 2017); conectando el contexto profesional con el aula mediante actividades de aprendizaje situado (Pan, 2016), y relacionando ambos ámbitos de manera más estrecha, como es el caso de las mentorías (Olalla-Soler, 2018).

Asimismo, un punto clave del desarrollo en la formación de intérpretes y su futura emplea- 
bilidad son las inserciones laborales supervisadas (Schnell y Rodríguez, 2017), también conocidas como prácticas profesionales, dado que facilitan la integración del estudiante con la experiencia profesional real. Sin embargo, la realidad de las prácticas profesionales en la formación de intérpretes en Chile se distancia de una posición laboral en agencias de interpretación o de actividades externas supervisadas por la institución formadora. No obstante, esto no significa que los estudiantes carezcan de la posibilidad de ejercer como intérpretes en un contexto real previo a su graduación.

Para los fines de este trabajo, se entenderá como experiencia profesional toda actividad de interpretación real que el estudiante haya realizado dentro de su proceso formativo antes de su egreso o titulación, ya que estas actividades profesionales tienen el potencial de convertirse en mediaciones privilegiadas del proceso de formación de las identidades profesionales (Andreozzi, 2011). Además, estas instancias pueden dar luces sobre el mismo proceso formativo en relación con los conocimientos adquiridos durante su actividad profesional, así como también mejorar la malla curricular y las metodologías de aprendizaje y enseñanza, posibilitando la adquisición de conocimientos, habilidades y competencias, ya que no solo se aplica lo aprendido en la formación, sino que se adquieren nuevos conocimientos y estrategias para abordar problemas en situaciones reales, en ambientes por lo general complejos e inciertos, lo que promueve un desarrollo integral del estudiante (Chan-Pavon, Mena-Romero, Escalante-Euán y Rodríguez-Martín 2018; Villa y Poblete, 2007).

\section{Metodología}

A partir de los antecedentes y características mencionadas, se estableció la presente investigación como un estudio enmarcado en la tradición de carácter socioconstruccionista de tipo interpretativo-descriptivo. Así se pudo indagar en la manera como los sujetos perciben la experiencia profesional, sus expectativas y, principalmente, su evaluación de la formación que recibieron. Debido a que este estudio se enfoca en un grupo de participantes de diferentes instituciones, edades y género, esta investigación cualitativa se centra en la indagación de los mundos subjetivos de estos participantes (Glesne, 2006), buscando entender e interpretar cómo aquellos construyen y significan sus experiencias. El método cualitativo permite obtener una comprensión más profunda de las experiencias vividas por los participantes del estudio, y lograr una descripción más rica de su contexto, mediante el diálogo interactivo entre el investigador y el investigado. Así, se apunta a un proceso de promoción de las voces de los participantes incipiente en la carrera de interpretación en el marco del paradigma dialógico de la educación.

\subsection{Participantes}

El muestreo de participantes se realizó con el propósito de contar con una muestra manejable, que diera cuenta de diferentes perspectivas y realidades de los sujetos. Para ello se realizó una invitación en línea a los estudiantes de interpretación con experiencia profesional de la región de Valparaíso, Chile, a participar voluntariamente en la investigación. Esto se hizo en dos momentos diferentes. El primero, en mayo y junio de 2017, convocó a nueve participantes y, en diciembre de 2017, se sumaron seis participantes. Todos ellos accedieron a la entrevista, que se llevó a cabo en las dependencias universitarias de cada estudiante, luego de la firma del correspondiente consentimiento informado. La mayoría de los participantes consiguieron la experiencia profesional a través de un docente y realizaron diversas técnicas de interpretación, información que se resume en la Tabla 1, junto 
Tabla 1. Participantes

\begin{tabular}{lllllll}
\hline Estudiante & Edad & Sexo & Semestre & Institución & Contacto & Tipo de interpretación \\
\hline 1 & 24 & Masculino & Octavo & PUCV & Profesor & Simultánea \\
\hline 2 & 22 & Femenino & Octavo & PUCV & Profesor & Bilateral consecutiva \\
\hline 3 & 22 & Femenino & Octavo & PUCV & Profesor & Bilateral y consecutiva \\
\hline 4 & 21 & Femenino & Sexto & PUCV & Profesor & Simultánea y bilateral \\
\hline 5 & 23 & Femenino & Octavo & PUCV & Profesor & Simultánea \\
\hline 6 & 22 & Femenino & Octavo & PUCV & Institución & Simultánea \\
\hline 7 & 22 & Femenino & Octavo & PUCV & Profesor & Simultánea \\
\hline 8 & 23 & Femenino & Octavo & PUCV & Profesor & Simultánea \\
\hline 9 & 22 & Masculino & Octavo & PUCV & Institución & Simultánea \\
\hline 10 & 23 & Femenino & Séptimo & UDLA & Profesor & Bilateral \\
\hline 11 & 24 & Femenino & Séptimo & UDLA & Profesor & Bilateral \\
\hline 12 & 27 & Masculino & Séptimo & UDLA & Externo & Simultánea y bilateral \\
\hline 13 & 25 & Femenino & Octavo & UPLA & Externo & Simultánea \\
\hline 14 & 24 & Masculino & Séptimo & UPLA & Externo & Simultánea y bilateral \\
\hline 15 & 26 & Femenino & Octavo & UPLA & Profesor & Simultánea y bilateral \\
\hline
\end{tabular}

con el semestre que cursaban los sujetos al momento de realizar la actividad.

\subsection{Recolección y análisis de datos}

Con el propósito de indagar en las percepciones y expectativas de los sujetos se realizaron quince entrevistas en profundidad semiestructuradas, las que luego fueron analizadas mediante la técnica de análisis de contenido, procedimiento que permite analizar y cuantificar los materiales de la comunicación humana mediante una estrategia de lectura interpretativa que pretende reemplazar un análisis somero de los datos recogidos, por uno más en profundidad, más productivo y rico con el fin de comprender, analizar e interpretar el contenido subjetivo de las entrevistas (Flick, 2004). De este modo se generaron unidades de análisis que se clasifican en categorías con el fin de proporcionar una representación simplificada de los datos, las que serán explicitadas más adelante.

Los datos fueron analizados a través del software Dedoose, programa en entorno web diseñado por académicos de la UCLA para desarrollar métodos de investigación mixta. Este software permitió un trabajo de análisis cualitativo en línea, ya que los datos se almacenan en una nube. De este modo, fue posible desarrollar el proyecto a distancia entre los tres investigadores. En primera instancia se generaron códigos libres arrojados en los discursos de los sujetos, para luego agruparlos en códigos de segundo orden. Estos, a su vez, se clasificaron en diferentes categorías que dieron paso a núcleos de análisis. Así, el análisis realizado es, en primera instancia, descriptivo-fenomenológico, ya que se pretende describir los núcleos creados, para luego dar paso a un análisis de carácter hermenéutico, dado que se introducen las guías inter- 
pretativas sobre las que se sustenta el análisis y la discusión de los hallazgos.

\section{Resultados}

Tras la categorización libre fue posible encontrar una serie de conceptos sensibles, representados por 181 códigos libres de información que fueron categorizados en 3 núcleos de análisis: expectativas, experiencia y formación. Si bien el objetivo del estudio es indagar en la percepción y expectativa que tienen los estudiantes con experiencia profesional respecto a su formación, se explicitaron diversas líneas argumentativas en los relatos recogidos, por lo que fue necesario aislarlos y categorizarlos.

\subsection{Expectativas respecto a la experiencia profesional}

En primer lugar, las expectativas de los estudiantes respecto a su carrera profesional corresponden a las creencias generalizadas que tienen los sujetos sobre su futuro laboral como intérpretes, las que fueron clasificadas en dos momentos: antes de la experiencia profesional y después de esta (Figura 1). Dicha distinción se debe a que las expectativas se crean y cambian según la experiencia directa o indirecta, la observación, persuasión verbal y el estado fisiológico (Peralta, 2006).

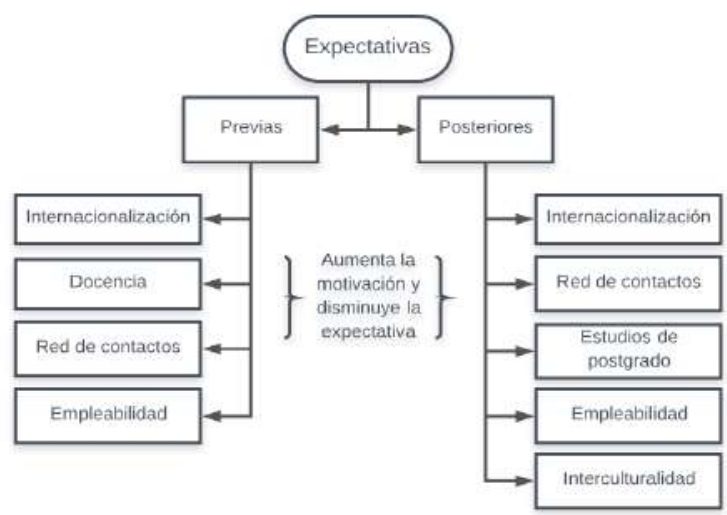

Figura 1. Núcleo de análisis: expectativas sobre la experiencia profesional
Como se puede apreciar en la Figura 1, previo a la experiencia profesional los sujetos señalaron de manera bastante vaga y sencilla que su primer objetivo era conseguir trabajo y crear una red de contactos profesionales. Al mismo tiempo, dos de ellos apuntaron que Naciones Unidas era una de las opciones que barajaban para su futuro entorno profesional, al igual que la docencia universitaria.

Al ser consultados por sus expectativas profesionales después de la experiencia (Tabla 2), se pudo evidenciar que la motivación de los sujetos por ser intérpretes aumentó, pero al mismo tiempo, las expectativas respecto a su futuro disminuyeron. Desde los relatos se desprende que existe un grado de incertidumbre al hablar sobre su futuro profesional, específicamente por las dificultades de encontrar un trabajo estable como intérprete, dado el contexto que conocieron durante sus experiencias profesionales.

Tabla 2. Expectativas profesionales de los sujetos.

Entrar al mundo de la interpretación me pare-

- ce que ya queda solamente para las perso-

Ð nas que hacen muy buenas interpretaciones o que ya han hecho interpretaciones y que son profesionales. Entonces, me parece que es un poco complicado entrar al mundo de la interpretación.

He escuchado experiencia de, por ejemplo, excompañeros, de nuestros mismos profesores, de que les costó encontrar trabajo y que obviamente uno tiene que buscar, uno tiene que ofrecerse. Por eso decidí irme para Santiago, porque yo creo que allá voy a encontrar quizás algo más, no más rápido, pero sí voy a encontrar quizás más.

Ahora me gustaría buscar un trabajo donde * pueda interactuar con las personas que vienen de otros países, que tienen distintas culturas. En realidad, siempre ha sido ese mi objetivo y tratar de sacar algún magíster o postgrado en algo que tenga que ver con cultura. 


\subsection{Percepción de la experiencia profesional}

En cuanto a la experiencia profesional en sí, todos los sujetos tienen una percepción positiva. La percepción corresponde al juicio que se elabora en torno a las sensaciones obtenidas del ambiente físico y social donde intervienen procesos psíquicos, como el aprendizaje, la memoria y la simbolización. Por supuesto, se entiende que la percepción del sujeto es subjetiva, selectiva y temporal (Vargas, 1994). En este sentido, lo positivo de la experiencia no solo se debe a que adquirieron nuevos conocimientos, sino que también comprendieron que contar con una actividad extracurricular es de mucha utilidad en sus futuros profesionales (Tabla 3).

Se aprecia, en primera instancia, que la evaluación positiva de los sujetos sobre la experiencia depende del resultado de la misma, es decir, la totalidad de los entrevistados sostiene que, a pesar del nerviosismo, el estrés y los posibles errores cometidos, tuvieron un buen rendimiento durante la interpretación, lo que resultó en un aumento de la motivación.

Tabla 3. Percepción de la experiencia profesional.

\begin{tabular}{|c|c|}
\hline & $\begin{array}{l}\text { La experiencia misma, la calle. Trabajar a } \\
\text { aprender. Por mucho que veamos videos, ensa- } \\
\text { yos, estar en una conferencia es distinto a estar } \\
\text { en la sala. Hay cosas que por mucho que las } \\
\text { diga un profesor, no las vamos a saber hacer } \\
\text { hasta que lo hagamos. }\end{array}$ \\
\hline & $\begin{array}{l}\text { Fue súper útil. Me motivó, me ayudó. Hay cosas } \\
\text { que nos han dicho los profes, que hemos visto } \\
\text { en clases también, pero hacerlo en un entorno } \\
\text { real, con gente que depende de ti es diferen- } \\
\text { te. La presión es diferente pero al mismo tiempo } \\
\text { estimulante. }\end{array}$ \\
\hline
\end{tabular}

En las entrevistas se indagó la aplicación de conocimientos aprendidos en su formación. No obstante, los relatos arrojaron también conocimientos aprendidos durante la experiencia profesional (Figura 2).

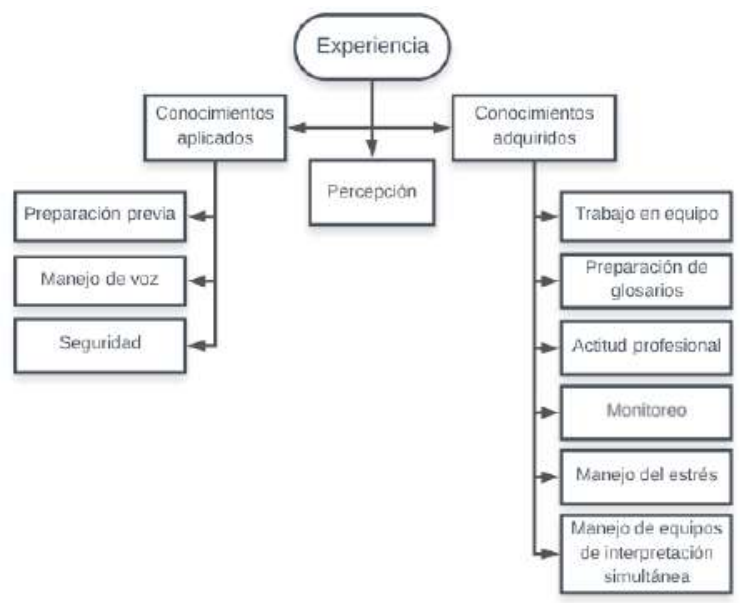

Figura 2. Núcleo de análisis: percepción de la experiencia profesional

En términos generales se observa que los sujetos ciertamente poseen el conocimiento previo que se necesita para llevar a cabo una preparación adecuada en lo que respecta una interpretación real. Es importante, entonces, señalar que dentro de los conocimientos aplicados la preparación previa es la fase más importante, y abarca las distintas formas en las que se pueden preparar para una interpretación. Sin embargo, también se declara la posibilidad de que la preparación puede ser nula debido a la falta de tiempo para hacerlo o a situaciones inesperadas durante la interpretación. Dentro de los métodos para prepararse para una interpretación se encontraron la búsqueda terminológica, la búsqueda de información sobre el orador y la traducción de documentos. Al mismo tiempo se da énfasis en los elementos prosódicos del lenguaje como la entonación, el tono de voz, las expresiones faciales, las pausas y la seguridad al entregar el mensaje, cuya preparación se hizo mediante la práctica con videos de los oradores o de temas similares (ver Tabla 4).

En cuanto a la experiencia y los conocimientos adquiridos en ella (Tabla 5), se destaca la actitud profesional y el manejo del estrés. La ansiedad generada a partir de la posibilidad de interpretar en un contexto real afectó a los su- 
Tabla 4. Relatos sobre los conocimientos aplicados.

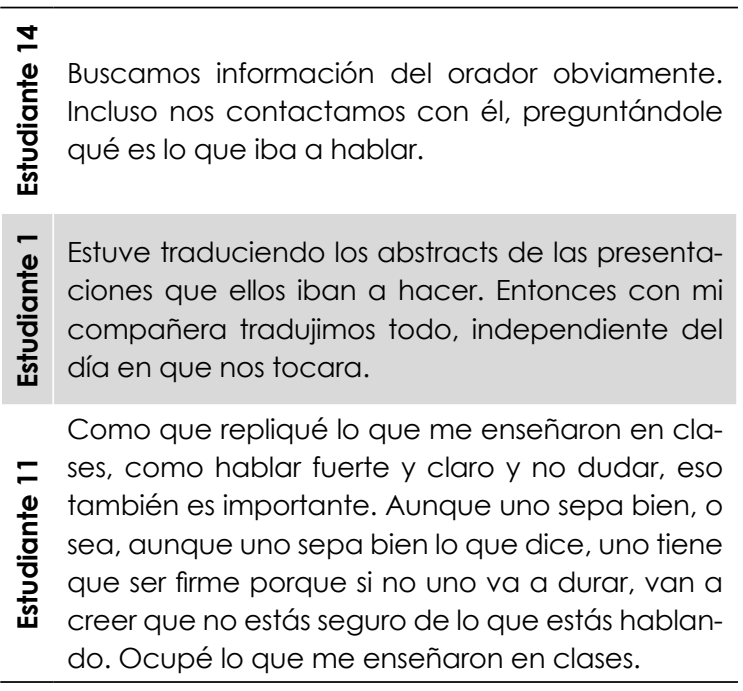

jetos. Todos declararon haberse sentido muy nerviosos antes de comenzar la interpretación. Pese a esto, se señala en los relatos que una vez comenzada la actividad, junto con el hecho de que en varios casos sus profesores fueron sus compañeros de cabina, pudieron sentirse más cómodos, apoyados, confiados. Junto a esto, también declararon que la actitud profesional y el contacto con los clientes son aspectos relevantes que no habían considerado durante su formación. Además de aprender a manejar diferentes equipos de interpretación simultánea, los sujetos hicieron hincapié en la relevancia que tiene la claridad y calidad de su mensaje, por lo que tuvieron que usar técnicas de monitoreo constantes.

\subsection{Formación de intérpretes}

El último núcleo de análisis corresponde a las expectativas y la percepción que tienen los estudiantes sobre su formación; son comentarios enfocados principalmente en los métodos de evaluación, las instalaciones de cada casa de estudio, críticas a las mismas, metodologías de aprendizaje y enseñanza, didáctica y los docentes (Figura 3).
Tabla 5. Relatos sobre los conocimientos aprendidos.

Un día te van a llamar a ti y luego a otra persona

que salió dos años antes que tú, ¿̇cachai? [sic]

Entonces antes tenía mis compañeros de cabina y ahora creo que me llevo muchos mejor con los demás, porque esto me ha servido a desplegar-

me, porque quizás nos toca trabajar juntos más adelante.

Ahora sé lo que es estar en una interpretación real, con gente que uno no conoce, con la presión de hacerlo bien, de darle una buena impresión al cliente, porque al final se trata de eso, de dejar una buena impresión con el trabajo que uno hace.

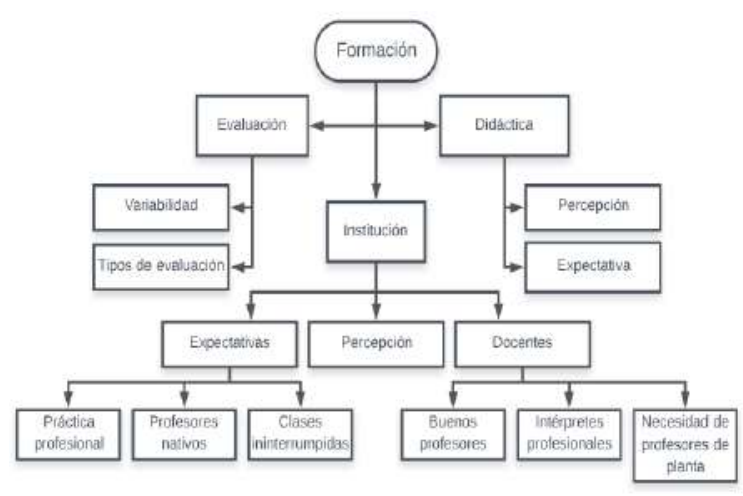

Figura 3. Núcleo de análisis: percepción de la experiencia profesiona

En primer lugar, los relatos señalan la amplia variabilidad en los métodos de evaluación aplicados en los procesos de formación (Tabla 6). Los sujetos indican que estos cambian incluso de un profesor a otro, cosa que no siempre facilitaba los aprendizajes, ya que asociaban los resultados de su evaluación con la subjetividad de cada docente. Aun así, la percepción es en general positiva, pues se destacan diferentes tipos de evaluaciones preferidas, como las mock conferences y los videos de conferencias. No obstante, vale destacar que se observa en cuatro relatos una práctica que molesta a los estudiantes con experiencia profesional por alejarse demasiado de la realidad laboral de la interpretación. Se refieren específicamente a evaluaciones de interpretaciones hechas sobre videos entregados con anterioridad, teniendo 
tiempo para escucharlo repetidas veces y preparar lo que se va a decir.

Tabla 6. Relatos sobre la evaluación.

Las evaluaciones dependen de cada profesor. Hay algunos que piden grabaciones para des-

으 pués escucharlas. Hay otros que están ahí en la

cabina con nosotros y nos están escuchando siempre, pero todos nos dan consejos que ellos aprendieron en sus años de trabajo o de sus mismos profesores. Es como una cadena que se va traspasando.

[...] que no te preparen para eso (hablando de evaluación de interpretación simultánea). No escucharlo antes para no aprendérselo de memoria ni nada. Llegar e ir como en la realidad.

En cuanto a la didáctica se evidencia una percepción ambivalente. Los sujetos declararon que hubo un cambio en sus expectativas y la percepción que tenían de su proceso formativo. Por una parte, los relatos señalan que uno de los aspectos positivos de su formación es la práctica constante, con situaciones reales de interpretación, ya sean conferencias hechas por los mismos estudiantes, por el profesor o con invitados externos, junto con la relevancia del trabajo en cabina y en grupo. Estas situaciones reales contribuyeron positivamente en su experiencia profesional, ya que se sintieron más preparados. Sin embargo, al mismo tiempo los sujetos declaran puntos negativos, como la falta de cursos dedicados al aprestamiento laboral, la gestión e incluso manejo de finanzas, considerando que en la carrera predomina la modalidad de trabajo independiente. A su vez se señala que en ocasiones falta más práctica de la interpretación aun, en sus distintas modalidades, incluyendo la interpretación inversa.

Siguiendo esta misma línea, los relatos también dan luces de nuevas expectativas respecto a su formación, sobre cambios o modificaciones que aplicarían en el aula, considerando lo vivido en sus experiencias profesionales (Tabla 7). Cada uno afirma de manera subjetiva lo que esperan obtener de su formación, señalando que el contenido teórico es importante, ya que sirve como el cimiento sobre el cual se construyen nuevos aprendizajes que luego se aplican en la práctica. Se destaca una vez más la importancia de tener prácticas en vivo, con oradores reales, más que solo videos. Además, se menciona la importancia de contar con interpretación inversa en los cursos, dado que en las experiencias vividas se les demandó en su mayoría interpretaciones inversas.

Tabla 7. Percepciones y expectativas de la didáctica

- Durante el semestre hicimos un poco de todo.

Practicamos mucha simultánea, porque tenemos

흐 la cabina este semestre, hicimos consecutiva en

terreno una vez y también hemos hecho sight translation. Eso ayuda mucho.

Yo creo que hace falta incorporar más formación

๘ en cuanto les comentaba recién: trato con el

б cliente [...] más de comportamiento profesional

que se refleja en lo que los profes no cuentan de

sus experiencias y cómo actúan y lo que tienen que hacer, pero no es un curso formal.

$\frac{m}{0}$
$\frac{0}{5}$
$\frac{0}{0}$
$\frac{1}{4}$

Se deberían mejorar detalles muy finos. Me gustaría que hubiera más prácticas en vivo.

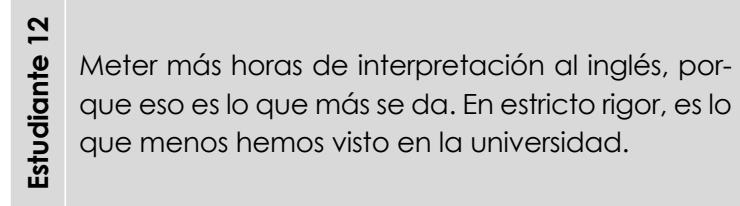

A nivel institucional, los relatos dan cuenta de una percepción positiva y negativa en cuanto a infraestructura. Unos señalan que los equipos son suficientes y adecuados, mientras que otros mencionan la falta de más equipamiento para interpretación. Por otro lado, también se hace referencia a un problema en la malla curricular, donde el Estudiante 15 hace referencia a la complicación que surge al no tener una 
continuidad en la práctica de interpretación, ya que los cursos al respecto se terminan de dictar mucho antes que los cursos de traducción. Vale mencionar que, de las tres instituciones mencionadas en este estudio, la PUCv es la única que cuenta con una carrera exclusiva de interpretación inglés-español. La UDLA y la UPLA ofrecen el título de traductor-intérprete. Junto a esto, también un estudiante hace referencia a la necesidad de contar con profesores nativos en su programa y cuatro sujetos también mencionan su molestia con la frecuencia con la que ocurren paros en sus instituciones.

Considerando lo anterior, lo más relevante es que la mayoría de los sujetos, independientemente de la institución, expresaron la necesidad de que los programas cuenten con una práctica profesional externa (Tabla 8). Si bien dan cuenta de que realizar una práctica profesional en el mercado laboral de la interpretación puede ser complejo, mencionan la relevancia que esta tendría a los estudiantes de la interpretación, desde el punto de vista formativo, pero también profesional.

Tabla 8. Percepciones y expectativas sobre la práctica profesional

Es distinto, es más real. Es distinto estar en el ambiente seguro de la universidad, de la cabi-

$\mp$ na con los profesores que ya conocemos. Es la

¿ misma burbuja en la que hemos estado siempre, cuatro años. La universidad debería hacerse más cargo de las prácticas. Quizás es algo utópico, porque si lo pensamos es súper difícil encontrar una práctica fuera de la universidad.

\& Es difícil hacer una práctica de interpretación đ. porque la interpretación no puede ser práctica.

흐 A menos que sea en algún ámbito que quizás no ¿- es tan, no sé cómo decirlo para que no suene 岀 feo, pero que no sea tan oficial.

- La interpretación que hice me dio herramientas y y me mostró cosas que las clases no. Así que sí 흠 creo que es necesario hacer una práctica profesional interpretando en eventos reales, con gente que uno no conoce.
Siguiendo esta misma línea, los estudiantes también destacan el rol de los docentes como parte fundamental de su proceso de aprendizaje. Reconocen a sus profesores como pilares indispensables para formar una base de aprendizajes y experiencias que luego podrán aplicar en su formación y trayectoria profesional. Asimismo, destacan el hecho de que los profesores sean a la vez intérpretes activos en el mercado laboral, dado que les entrega no solo un nexo con la industria, sino que también comparten las experiencias profesionales que pueden, en ocasiones, parecer distantes de la academia. Junto a esto, también hay espacio para críticas, ya que se menciona la necesidad de contar con más profesores de planta en las instituciones (Tabla 9).

Tabla 9. Relatos sobre los docentes.

[Los profesores] tienen que estar activos. Tienen

ㅇ que saber cuáles son las técnicas nuevas. Si no

¿ las hay. Y él tiene que estar enterado de todo eso. Y eso es lo que me gusta de este profe, porque pasa en terreno. A veces no tenemos clases porque pasa en terreno haciendo interpretaciones y todo.

Se nota que los profesores saben de pedago-

Ð gía, que se han capacitado en eso para poder enseñar mucho mejor. A veces nos cuentan cómo se enseñaba antes la interpretación, como a ellos les enseñaron y es mucha la diferencia a las clases que tenemos.

\section{Discusión y conclusiones}

A raíz de los resultados obtenidos de los relatos, es posible señalar que la experiencia profesional en un contexto de interpretación real se transformó en una situación de aprendizaje para los sujetos, independiente del currículo universitario del que son parte. La formación práctica de un estudiante universitario es relevante no solo para el desempeño de la profesión, sino también para su desarrollo personal y académico (Raposo y Zabalza, 2011); así lo demuestran los relatos recogidos. 
En una primera instancia, la experiencia profesional generó expectativas en los sujetos. La psicología cognitiva explica este fenómeno señalando que todo comportamiento está dirigido a una meta, por lo mismo, las experiencias son razonadas, categorizadas y transformadas (Peralta, 2006). En el caso de los sujetos de este estudio, sus expectativas se vieron, hasta cierto punto, disminuidas, como por ejemplo en la opción de trabajar para organismos internacionales como la Organización de las Naciones Unidas. Al mismo tiempo, la preocupación que genera la posibilidad de encontrar un trabajo estable como intérprete aumenta en los sujetos tras la experiencia profesional, lo que no es exclusivo del mercado de la interpretación. La expansión de la educación superior ha hecho que la transición de la universidad al mercado laboral se dificulte debido a mayores cohortes de estudiantes buscando un primer trabajo (Salas-Velasco, 2007). En el contexto chileno, el mercado de la interpretación es estacional, con un aumento en la actividad durante el cuarto trimestre del año con temáticas que giran en torno a la medicina, la ciencia, la tecnología y la minería (Díaz-Galaz, 2017), áreas que se relacionan con la economía nacional dependiente de las importaciones de fuentes de energía y la exportación de materias primas. Por ello, la actividad profesional de los intérpretes en Chile se mueve en este entorno de seminarios, conferencias y reuniones corporativas e internacionales (Díaz-Galaz, 2017).

Al contar con una experiencia profesional fuera de la universidad, los sujetos entrevistados pudieron conocer de primera fuente la realidad dinámica $\mathrm{y}$, a veces, inestable del mercado de la interpretación. Sin embargo, al contrario de sus expectativas, su motivación aumentó. Así se crea cierta paradoja en la que, a pesar de que el mercado laboral es inestable y complejo, conocerlo aumenta la motivación, siem- pre mediado por este puente entre la teoría y la práctica del aula y la demanda del mundo profesional, así como también la búsqueda de estudios de posgrado. Esta paradoja puede encontrar su explicación en la psicología.

La perspectiva cognitiva sobre la motivación señala que el sistema cognitivo envía información a otros sistemas, como el afectivo, el comportamental y el fisiológico (Naranjo, 2009). Así, los pensamientos inciden en la motivación de las personas, y está influenciada por lo que pueden controlar en su ambiente, las metas y el monitoreo de su progreso (Naranjo, 2009). Considerando esto, el aumento de la motivación en los sujetos de esta investigación podría nacer del hecho de que las personas se motivan a realizar cosas y esforzarse para alcanzar una meta, por el valor asignado al resultado de su esfuerzo. Al tratarse de una primera instancia profesional, los sujetos, como señalan los datos, asignaron un alto valor a la experiencia, a pesar de que disminuyeran sus expectativas. Junto a esto, los relatos también señalan que la experiencia profesional les dio contacto con una red de profesionales y aumentó su conocimiento del mercado de trabajo, elementos que apoyan la consecución de sus metas.

En base a lo anterior los estudiantes calificaron la experiencia profesional de manera positiva, especialmente porque junto con ser una oportunidad para desempeñarse profesionalmente, también es una instancia de aprendizaje situado en escenarios reales, lo que hace posible no solo la aplicación de los conocimientos aprendidos durante la formación, sino también la adquisición de nuevos saberes, habilidades y competencias necesarias para el ejercicio profesional (Chan-Pavon, 2018). Al mismo tiempo, los relatos hacen hincapié en el rol del intérprete en contextos interculturales, lo que se configura como un paso más en 
la consolidación de una identidad profesional. En este sentido, la identidad no es algo que se tenga, sino que se desarrolla y se describe mediante la negociación con diferentes entornos y situaciones (Beijaard, Meijer, Verloop, 2004). Es así como este proceso se nutre de todo tipo de experiencias, como la situación de aprendizaje experiencial directo vivida por los sujetos de esta investigación, lo que permite forjar su identidad profesional uniendo el hacer y el ser, apuntando a que el estudiante no solo sea actor, sino también activista de su formación (García, González y Martín-Cuadrado, 2016).

Lo anterior guarda relación con el compromiso que muestran los sujetos con su propia formación después de la experiencia profesional, pues pueden ser más críticos con ella y así contribuyen a su mejora. E1 compromiso estudiantil es más que el involucramiento o la participación; requiere también de la sensación de que las cosas tienen un sentido (Harper y Quaye, 2009) que es precisamente el componente que otorga la experiencia profesional vivida.

De la mano con lo anterior, son los mismos relatos de los entrevistados los que demandan una instancia formal de práctica profesional con características similares a la experiencia vivida por ellos, destacando que este proceso es de suma importancia en la adquisición y aplicación de conocimientos y en el desarrollo y consolidación de competencias, lo que también se aplica al ámbito de la interpretación, ya que no solo mejora la empleabilidad de los graduados (Schnell y Rodríguez, 2017), sino que, al igual que las mentorías, favorece los resultados actitudinales, relacionales y motivacionales de los estudiantes, junto con la motivación y promoción de diferentes habilidades y satisfacciones personales (Olalla-Soler, 2018).
Los resultados cualitativos de la muestra apuntan a que la experiencia profesional es multidimensional, aportando en áreas como la evaluación, la didáctica y el currículo. La participación estudiantil en temáticas relacionadas con los contextos universitarios puede ser crítica para el desarrollo de nuevas políticas educativas que apunten a la mejora de los procesos de aprendizaje y enseñanza (Bovill, Cook-Sather y Felten, 2011).

Del mismo modo, la percepción que tienen los sujetos respecto a los docentes marca un antecedente valioso, ya que no solo se reconoce la capacidad de los profesores para generar un proceso formativo adecuado, sino que también la experiencia profesional apunta a una resignificación de la relación docente-alumno en el campo de la interpretación. Existe evidencia suficiente para asegurar que las colaboraciones entre estudiantes y docentes estimulan una revisión de las relaciones alumno-profesor, junto con las responsabilidades de su aprendizaje (Cook-Sather, 2007), y esto deriva en una mejora en la calidad de la educación. El rol de los estudiantes provee un reconocimiento de las complejas realidades de poder y a su vez estimula la creación de un componente que Polman y Pea (2001) denominaron comunicación transformativa, definida como el proceso social que permite a docentes y alumnos crear significados que, por separado, no pueden considerar, lo cual estimula una pedagogía abierta y centrada en el estudiante.

De este modo, se puede deducir, a partir de los resultados, que la horizontalidad generada en las diferentes experiencias profesionales, en las que profesores y estudiantes en su mayoría fueron compañeros de cabina, lleva a amplificar las voces del alumnado, configurando una mayor participación en su proceso educativo como cocreadores del proceso de enseñanza, del diseño de programas y del currículo. 
Al mismo tiempo, los relatos destacan el importante rol de los profesores en el proceso de transición de los estudiantes de interpretación de la universidad al mercado laboral, dado que, como intérpretes activos en el contexto profesional de la interpretación, los docentes son la mayoría de las veces el primer enlace de una cadena de contactos que puede dar paso a una carrera sustentable como intérprete.

En conclusión, la indagación en las experiencias profesionales de los estudiantes de interpretación se configura como una herramienta de reflexión en diversos aspectos de la formación de intérpretes. La riqueza que ofrecen las subjetividades de los entrevistados permitió abarcar una gran variedad de temas relacionados con el proceso de enseñanza y aprendizaje, en el que la voz de los estudiantes con experiencia profesional, de la mano con la motivación y el compromiso de todas las partes de la comunidad educativa, pueden conformarse como la base de un aprendizaje centrado en el estudiante, ya que sin motivación no existe el impulso de aprender y sin voz no existe la autenticidad en los procesos de aprendizaje y enseñanza. Por ello, los hallazgos revelan una riqueza de aportes derivados de la experiencia profesional de los estudiantes, previa a la graduación. Esos aportes y el nuevo lugar que puede asumir el estudiante como actor de su proceso formativo se inscribe en el paradigma dialógico de la educación, que se mencionó al inicio. Eso sustenta la pertinencia de este tipo de iniciativas en la formación de intérpretes.

\section{Referencias}

Álvarez-Álvarez, S. y Arnáiz-Uzquiza, V. (2017). Translation and interpreting graduates under construction: do Spanish translation and interpreting studies curricula answer the challenges of employabil- ity? The Translator and Interpreter Trainer, 11(2-3), 139-159.

Andreozzi, M. (2011). Las practices profesionales de formación como experiencias de pasaje y tránsito identitario. Archivos de Ciencias de la Educación, 5(5), 99-115. Recuperado de: http://www.memoria. fahce.unlp.edu.ar/art_revistas/pr.5431/ pr.5431.pdf

Barik, H. (1971). A description of various types of omissions, aditions, errors of translation encountered in simultaneous interpretation. Meta, 16(4), 199-210.

Beijaard, D., Meijer, P. y Verloop, N. (2004). Reconsidering research on teachers' profesional identity. Teaching and Teacher Education, (20), 107-128. Recuperado de: http://bit.do/ePYRk

Bovill, C. Cook-Sather, A. y Felten, P. (2011). Changing participants in pedagogical planning: Students as co-creators of teaching approaches, course design and curricula. International Journal for Academic Development, 16(2), 133-145. DOI: https:// doi.org/10.1080/1360144X.2011.568690

Cabrera, T. (2017). The translation and interpreting industry in the United States. Informes del Observatorio / Observatorio's Report, (59). Recuperado de: http://cervantesobservatorio.fas.harvard.edu/sites/default/files/028_report_translation_2.pdf

Chan-Pavon, M., Mena-Romero, D., Escalante-Euán, J. y Rodríguez-Martin, M. (2018). Contribución de las prácticas profesionales en la formación de los estudiantes de la facultad de Ingeniería Química de la Universidad Autónoma de Yucatán (México). Formación Universitaria, 11(1), 53-62. DOI: http://dx.doi.org/10.4067/ S0718-50062018000100053

Choi, J. (2004). A metacognitive approach to evaluating consecutive interpretation for novice learners. Conference Interpreta- 
tion and Translation, 2(2), 169-185. DOI: https://doi.org/10.7202/013256ar

Coates, H. (2005). The value of student engagement for higher education quality assurance. Quality in Higher Education, 11(1), 25-36. doi: https://doi. org/10.1080/13538320500074915

Collados, A. y Fernández, M. (2001). Manual de interpretación bilateral. Granada: Comares.

Cook-Sather, A. (2007). Resisting the impositional potential of student voice work: lessons for liberatory educational research from poststructuralist feminist critiques of critical pedagogy. Discourse, 28(3), 389-403. DoI: https://doi. org/10.1080/01596300701458962

Correa, C. y Pereira, M. (2012). Internship experiences: Contributions to the school-to-work transition. Paidéia, 23(54), 102-112. Recuperado de: http://www. scielo.br/scielo.php?script $=$ sci_arttext\&pid=S0103-863X2013000100103\&1$\mathrm{ng}=\mathrm{pt} \& \mathrm{t} \operatorname{lng}=\mathrm{pt}$

De Miguel, J. M. (2007). Acreditación de educación superior. Madrid: CIs.

Delors, J. (1996). La educación encierra un tesoro. Madrid: Santillana-Ediciones UNESCO.

Díaz-Galaz, S. (2017). Formación de intérpretes e investigación en interpretación en Chile: desafíos y oportunidades para el desarrollo de los estudios de interpretación. Mutatis Mutandis, 10(2), 46-63. DOI: https://doi.org/10.17533/udea.mut. v10n2a02

Domínguez, E. (2016). Interpretación simultánea: el camino hacia la profesionalización [Tesis de pregrado]. Universitat Autònoma de Barcelona, Barcelona. Recuperado de: https://ddd.uab.cat/pub/ tfg/2016/tfg_44732/TFG_2015-16_FTI_ DOMINGUEZHERNANDO.pdf

Domínguez, L. (2015). La evaluación para el aprendizaje de la interpretación de conferencias. Concepciones y prácticas de docentes $y$ discentes en tres cursos de posgrado de Cataluña, Portugual y Canarias [Tesis doctoral]. Universitat Autònoma de Barcelona, Barcelona. Recuperado de: https://www.tdx. cat/bitstream/handle/10803/317965/ lda1de1.pdf?sequence $=1 \&$ isAllowed $=y$

Driesen, C. (1999). Francesca Gaiba. The origins of simultaneous interpretation. The Nuremberg Trial. Interpreting, 4(2), 223-224

Flick, U. (2004). Introducción a la investigación cualitativa. Madrid: Morata.

García, S., González, R., y Martín-Cuadrado, M. (2016). Influencia de las prácticas en el desarrollo de la identidad profesional de los estudiantes de educación social. Pedagogía Social. Revista Interuniversitaria, (28), 245-259. Recuperado de: https://www. redalyc.org/pdf/1350/135047100019.pdf

Gile, D. (1995). Basic concepts and models for interpreter and translator training. Philadelphia: John Benjamins.

Gile, D. (2005). Teaching conference interpreting: a contribution. En M. Tennent, (Ed.), Training for the new millenium (pp. 127-151). Amsterdam/Philadelphia: John Benjamins.

Gillies, A. (2005). Note-taking for consecutive interpreting. A short course. Manchester: St. Jerome.

Glesne, C. (2006). Becoming qualitative researchers: an introduction. New York: Pearson Education, Inc.

Harper, S. y Quaye, J. (2009). Student engagement in higher education. New York: Routledge.

Iglesias, E. (2007). La didáctica de la interpretación de conferencias: teoría y práctica. Granada: Comares.

Jabif, L. (2007). La docencia universitaria bajo enfoque por competencias. Valdivia: Imprenta Austral.

Jiménez, M. (2012). Primeros pasos hacia la interpretación inglés-español. Madrid/Castelló de la Plana: Universitad Jaume I, Servei de Comunicació i Publicacions. Edelsa. 
Jones, R. (1998). Conference interpreting explained. Machester: St Jerome.

Kalina, S. (2005). Quality assurance for interpreting processes. Meta, 50(2), 768-784. DOI: https://doi.org/10.7202/011017ar

Kiraly, D. (2000). A social constructivist approach to translator education: Empowerment from theory to practice. Manchester: Routledge.

Lee, Y. (2005). Self-assessment as an autonomous learning tool in an interpretation classroom. Meta, 50(4). Recuperado de https://www. erudit.org/fr/revues/meta/2005-v50-n4meta1024/019869ar.pdf

Mackintosh, J. (1999). Interpreters are made not born. En I. Kurz y M. Bowen (Eds.), History of interpreting (vol. 4, pp. 67-80). Amsterdam: John Benjamins.

Mihans, R., Long, D., y Felten, P. (2008). Power and expertise: Student-faculty collaboration in course design and the scholarship of teaching and learning. International Journal for the Scholarship of Teaching and Learning, 2(2), 1-9. DOI: https://doi. org/10.20429/ijsot1.2008.020216

Mungaray, L. (2001). La educación superior y el mercado de trabajo profesional. REDIE. Revista Electrónica de Investigación Educativa, 3(1). Recuperado de: https://www. redalyc.org/pdf/155/15503104.pdf

Naranjo, M. (2009). Motivación: perspectivas teóricas y algunas consideraciones de importancia en el ámbito educativo. Revista Educación, 33(2), 153-170.

OCDE. (2012). Fostering quality teaching in higher education: Policies and practices. Institutional Management in higher Education. Recuperado de: https://www. oecd.org/education/imhe/QT\%20policies\%20and\%20practices.pdf

Olalla-Soler, C. (2018). Bridging the gap between translation and interpreting students and freelance professionals. the mentoring programme of the Professional Association of Translators and
Interpreters of Catalonia. The Interpreter and Translator Trainer, 13(1), 64-85. DOI: 10.1080/1750399X.2018.1540741

Orlando, M. (2016). Training 21st century translators and interpreters: At the crossroads of practice, research and pedagogy. Berlín: Frank \& Timme GmbH.

Pan, J. (2016). Linking classroom exercises to real-life practice: a case of situated simultaneous interpreting learning. The Translator and Interpreter Trainer, 10(1), 107-132. DOI: https://doi.org/10.1080/175039 9X.2016.1154346

Peralta, J. (2006). Rol de las expectativas en el juicio de satisfacción y calidad percibida del servicio. Límite. Revista de Filosofia y Psicología, 1(14), 195-214. Recuperado de: https://dialnet.unirioja.es/descarga/ articulo/2252855.pdf

Pöchhacker, F. (2004). Introducing Interpreting Studies. Londres: Routledge.

Polman, J. y Pea, R. (2001). Transformative communication as a cultural tool for guiding inquiry science. Science Education, 85, 223-238. DOI: https://doi.org/10.1002/ sce. 1007

Raposo, M. y Zabalza, M. (2011). La formación práctica de estudiantes universitarios. Repensando el practicum. Revista de Educación, 354, 17-20. Recuperado de: https://www.mecd.gob.es/ $\mathrm{dctm} / \mathrm{revista-de-educacion/numer-}$ os-completos/re354.pdf?documen$\mathrm{tId}=0901 \mathrm{e} 72 \mathrm{~b} 811 \mathrm{c} 5824$

Ruiz, L. y Diur, M. (2017). Employability in the United Nations: An empirical analysis of interpreter training and the LCE. The Interpreter and Translator Trainer, 11(2-3), 223-227. DOI: https://doi.org/10.1080/1 750399X.2017.1344921

Salas-Velasco, M. (2007). The transition from higher education to employment in Europe: the analysis of the time to obtain the 
first job. Higher Education, 54(1), 333-360. DOI: $10.1007 / \mathrm{s} 10734-006-9000-1$

Schnell, B. y Rodríguez, N. (2017). Ivory tower vs. workplace reality. The Interpreter and Translator Trainer, 11(2-3), 160-186. Dor: https://doi. org/10.1080/1750399X.2017.1344920

Seleskovitch, D. (1975). Langage, langues et mémoire: études de la prise de notes en interprétation consécutive. París: Minard.

Takeda, K. (2010). What interpreting teachers can learn from students: A case study. The International Journal for Translation \& Interpreting Research, 2(1), 38-47. Recuperado de: http://trans-int.org/index.php/transint/article/view/88

UNESCO (1998). La educación superior en el siglo XXI. Visión y acción. En Conferencia Mundial sobre la Educación Superior (tomo I). Informe final. Disponible en https://unesdoc.unesco.org/ark:/48223/ pf0000116345_spa

Vargas, L. (1994). Sobre el concepto de percepción. Alteridades, 4(8), 47-53.

Villa, A. y Poblete, M. (2007). Aprendizaje basado en competencias: Una propuesta para la evaluación de las competencias genéricas. Bilbao: Universidad de Deusto.

Walker, J. y Shaw, S. (2011). Interpreter preparedness for specialized settings. Journal of Interpretation, 21(1), 96-107. Recuperado de http://bit.do/ePYRy

Zabalza, M. (2009). Diseño y desarrollo curricular. Madrid: Narcea.

Zabalza, M. A. (2011). El prácticum en la formación universitaria: estado de la cuestión. Revista de Educación, (354), 21-43. Recuperado de http://www.revistaeducacion.educacion.es/re354/re354_02.pdf

Cómo citar este artículo: Pavez-Gamboa, P., Urra-Urtubia, Á. y Carvajal-Pallacán, C. (2019). Las experiencias profesionales de los estudiantes de interpretación como fuente de mejora en los programas de formación de intérpretes en Chile. Mutatis Mutandis. Revista Latinoamericana de Traducción, 12(1), 230249. DoI: https://doi.org/10.17533/udea.mut.v12n1a09 